\title{
Unusual location of molars called as kissing molars: A case report
}

\author{
${\text { Geeta Singh }{ }^{1, *} \text {, Dipti Daga }}^{2}$, U. Vignesh ${ }^{3}$, Rashmi Agrawal $^{4}$ \\ ${ }^{\mathbf{1}}$ Associate Professor, ${ }^{2}$ Senior Resident, ${ }^{3}$ Junior Resident, ${ }^{\mathbf{4}}$ Reader, Dept. of Oral and Maxillofacial Surgery, ${ }^{\mathbf{1 - 3}}$ King George's \\ Medical University, Lucknow, Uttar Pradesh, ${ }^{4}$ BBD University, Lucknow, Uttar Pradesh, India
}

*Corresponding Author:

Email: drgeetasinghkgmc@gmail.com

\begin{abstract}
Introduction: Kissing molars also called as "rosette formation", these are unusual impacted teeth with their occlusal surfaces are in close apposition and the roots pointing in opposite direction. These are extremely rare conditions actually about less than thirty cases has been reported since now in the literature.

Materials and Methods: This case report describes a patient who visited our outpatient clinic for pain over her left side of mandible for last one months. OPG was advised which showed unusual impacted left lower second and third molar relationship with their occlusal surfaces facing each other. Management by extraction of both second and third molars with proper surgical curettage of the site was done.

Results: Postoperatively marked relief in the pain for the patient. Minimal postoperative swelling was observed immediate postoperative, which subsides after 5 days and there is no postoperative paraesthesia reported with relation to inferior alveolar nerve branch of trigeminal nerve and marginal mandibular nerve branch of facial nerve.

Conclusion: Kissing molars are rare unusual pathological condition which needs proper treatment planning and management for proper surgical removal to prevent possible chances of postoperative paraesthesia with relation to inferior alveolar nerve branch of trigeminal nerve and marginal mandibular nerve branch of facial nerve. This report documents better surgical management of a patient with kissing molars.
\end{abstract}

Keywords: Kissing molar, Impaction, Rare case, Management.

\section{Introduction}

Kissing molars are rare unusual impacted teeth pathological condition in which impacted teeth occlusal surfaces contacting towards each other in a single follicular space and their roots are pointing in opposite directions ${ }^{4}$. Bilateral involvement of kissing molars also has been reported in the literature. The most commonly affected impacted teeth are mandibular and maxillary third molars, maxillary canine, second mandibular premolars and maxillary premolars, central incisors and very rarely second molars respectively. ${ }^{6}$ In this case report third and second molars are involved on the left side.

Kissing molars term was first given by Vanhoof in 1973, in which he reported a case with bilateral kissing molars in one of the mentally retarted patient. ${ }^{1,2}$ Multiple rosetting of the molars has also been reported, as in two of the four cases reported by nakamura et al; in 1991 in the patients of whom suffering from mucopolysaccharidoses but we had no reason to believe that in our patient had mucopolysaccharidosis as this was a solitary radiological finding. ${ }^{5}$ This association should be considered in uncertain cases to perform further investigation.

However aetiology and pathogenesis of this condition is remain unknown. According to several studies there is no gender predilection for this condition, and age range is reported between 10-50 years. ${ }^{1}$

In the present case report study, we reported kissing molar case between second molar and third molar teeth which is managed by surgical removal of both the teeth and surgical curettage of the extracted site.

\section{Case Report}

A 37-year-old male patient presented to our outpatient clinic, with the chief complaint of pain over left lower back region of jaw since 1 month. On recording history of his present illness, he was apparently asymptomatic 1 months back until he felt pain over his left back region of the lower jaw. Then he visited some nearby private dental clinic where he was managed conservatively with some analgesics medication but he didn't get relief.

On clinical examination, patient had tenderness over left side of angle region of mandible, but there is no swelling, and paraesthesia in the left lower jaw. On intraoral examination patient had missing left lower second and third molars teeth. No history of any extraction done before.

Then patient is advised for orthopantamogram, which shows unusual impacted second and third molar with occlusal surface facing each other and roots are pointing opposite to each other and distal root of second molar is appearing to be extend up to lower border of the mandible. Crowns of both the impacted molars are appearing to be present in single follicular sac and third molar is present above inferior alveolar canal and second molar is appearing to extend up to the lower border of mandible present buccally along inferior alveolar nerve canal. 


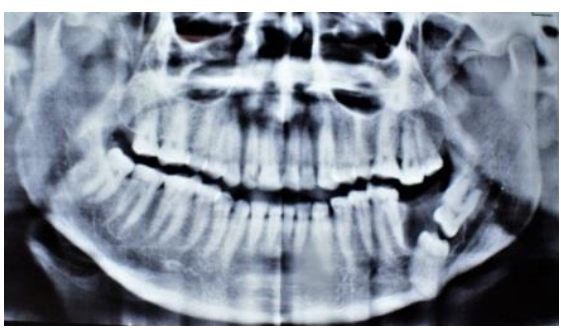

Fig. 1

Then patient was advised for surgical extraction of the teeth. As second molar is inferiorly placed along the lower border of the mandible, to minimize the chances of mandibular fracture, inferior alveolar nerve damage and minimum tissue trauma extra-oral submandibular approach was planned for direct access to the impacted teeth.

Surgical Management: After proper informed consent surgical extraction was done under local anaesthesia. A submandibular incision was given and then roots of second molar is exposed after careful buccal bone guttering. Roots of second molars are assessed and location related to inferior alveolar canal was properly assessed. Then carefully roots of second molar was splitted from crown at the cervical area of teeth and roots of second molar was elevated and extracted, which creates space for the removal of crown of the second molar. Then again some buccal bone is also removed for the exposure of crown of third molar which creates space for elevation and pulling of third molar along the same path from where the second molar was extracted. After extraction of second and third molar bony socket was curetted and proper toileting was done. Then layer by layer closure of the wound was carried out using 3-0 vicryl and 4-0 nylon suture material.

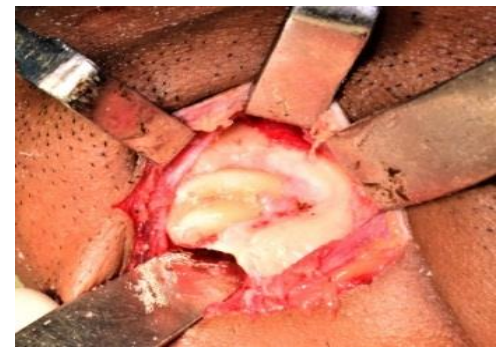

Fig. 2:

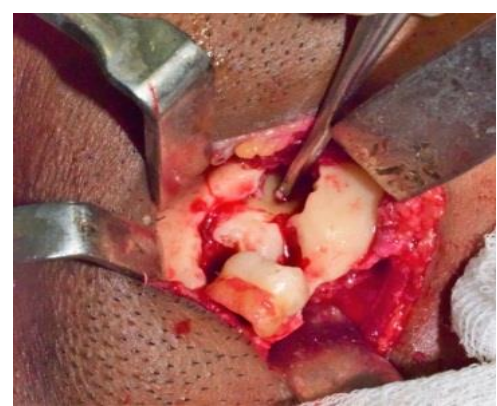

Postoperatively patient was prescribed for Inj. Cefaperazone + sulbactum $1.5 \mathrm{gm}$ i.v B.D, inj. Metronidazole $100 \mathrm{mg}$ i.v TDS, a non-steroidal drug (containing aceclofenac 100mg + Paracetomol 500mg + serratiopeptidase 15mg) 1 tab B.D, and tab. Rabeprazole $20 \mathrm{mg}+$ domperidone $30 \mathrm{mg} 1 \mathrm{tab}$ B.D for 5 days

Sutures were removed $7^{\text {th }}$ day postoperatively.

\section{Results}

Patient was assessed after 1 week, and 1 month, which shows no postoperative complications like paraesthesia of inferior alveolar nerve and marginal mandibular nerve and had minimal postoperative swelling which subsides within 5 days of surgery. Postoperative radiographs shows intact lingual cortical plate at the operated site and along lower border of mandible. Patient got complete relief from his pain and completely satisfied with the results.

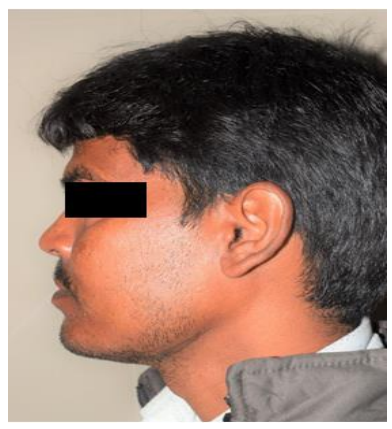

Fig. 4:

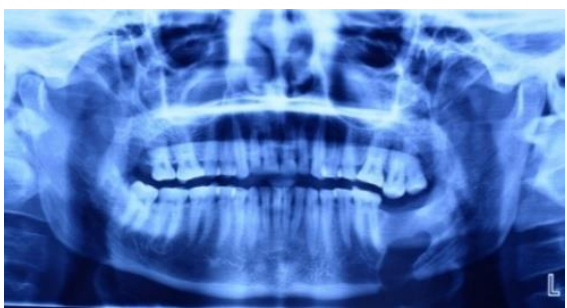

Fig. 5:

\section{Discussion}

Kissing molars are rare unusual pathological condition in which impacted teeth occlusal surfaces contacting towards each other in a single follicular space and their roots are pointing in opposite directions, this definition was given by Juneja in 2008 and Kissing molars term was first given by Vanhoof in 1973, in which he reported a case with bilateral kissing molars. Robinson et al in 1991 also described one of the similar type of case in which kissing molars was reported bilaterally in a younger patient of age 25, but doesn't described the management of this condition. Incidence of impacted third molars are reported in several studies but the incidence of second molar impaction was rarely reported. Preece in 1985 reported the prevalence of impacted second molars as only $0.03 \%$ from total of

Fig. 3: 
5000 cases he studied. The exact pathogenesis of this condition is still controversial. ${ }^{1,5}$

Multiple rosetting of the molars has also been reported, as in two of the four cases reported by Nakamura et al; in 1991 in the patients of whom suffering from mucopolysaccharidoses. ${ }^{5}$ In 1962, R.A Cawson reported a similar type of condition in a patient who is suffering from mucopolysaccharidosis (MPS) and suggested that MPS can be an etiological cause of this type of condition. ${ }^{2}$

In 2005, bakeen et al; reported a case of bilateral kissing molars in which lower third molar is associated with fourth or supernumerary molar teeth, and patient was managed by surgical extraction of all lower third and fourth molar bilaterally under general anaesthesia. ${ }^{2}$

In 2008, B. Krishnan reported a similar case in 35 yr. old female patient with kissing molars associated with dentigerous cyst in her left lower jaw which was managed surgically without any complications.

Similarly, In 2014 R.Z Sa Fortes et al; reported a case in which bilateral kissing molars associated with dentigerous cyst was shown in a $33 \mathrm{yr}$. old male patient, which was managed by surgical removal of teeth after planning with the help of preoperative $\mathrm{CBCT}$ and $\mathrm{OPG}$ X-rays. ${ }^{4}$

In 2016, T. zerener et al; reported a case report study in which they shown a 38 yr. old female patient with bilateral kissing molars which is managed surgically through intraoral approach, in which also shown that kissing molar is a rare unusual type of condition which needs proper treatment planning before extracting these molars, to prevent complications like inferior alveolar nerve paraesthesia, mandibular fractures. ${ }^{6}$

In most of the cases of kissing molars as reported in several studies in the literature, proper surgical management method was not clearly defined which makes management of this condition difficult. In our case report, we used extra-oral approach for surgical removal of this type of impacted condition, because in our patient roots of second molar was so inferiorly placed along lower border of the mandible and in close relation to inferior alveolar nerve canal. So extra-oral submandibular incision provided direct access to the impacted second molar which makes minimal bone removal during bone guttering so to prevent fracture of the mandible and also provide direct access to impacted teeth and shows better relation of inferior alveolar nerve with impacted teeth which helps in minimal damage of mandible and inferior alveolar nerve during surgery.

\section{Conclusion}

Kissing molars are rare unusual pathological condition which needs proper surgical treatment planning and management to prevent possible complications. This report documents better surgical management of a patient with this type of rare unusual impacted kissing molars.
Further studies needed with more number of cases, for explaining proper management protocol of this unusual rare condition.

\section{References}

1. Dardo Menditti, Luigi Laino, Marco Cicciù, Antonio Mezzogiorno, Letizia Perillo, Marco Menditti, Gabriele Cervino, Lorenzo Lo Muzio, Alfonso Baldi. Kissing molars: report of three cases and new prospective on aetiopathogenetic theories. Int J Clin Exp Pathol 2015;8(12):15708-15718.

2. Manuel Arjona-Amo, Eusebio Torres-Carranza, Antonio Batista-Cruzado, Maria-Angeles Serrera-Figallo, Santos Crespo-Torres, Rodolfo Belmonte-Caro, Claudio AlbisuAndrade, Daniel Torres-Lagares, José-Luis GutiérrezPérez. Kissing molars extraction: Case series and review of the literature. J Clin Exp Dent. 2016;8(1):e97-101.

3. Parveen Shahista, Rohan Mascarenhas, Sandeep Shetty, Akhter Husain. Kissing molars: An unusual unexpected impaction. Archives of Medicine and Health Sciences Jan-Jun 2013 / Vol 1 Issue 1.

4. Rafael Zago Sá Fortes, Valmor Salvaro Júnior, Filipe Modolo, Edson Mackowiecky. Kissing molars: Report of a case. Journal of Oral and Maxillofacial Surgery, Medicine, and Pathology 26 (2014) 48-51.

5. T. Nakamura, K. Miwa, S. Kanda, K. Nonaka, H. Anan, S. Higash and K. Beppu. Rosette formation of impacted molar teeth in mucopolysaccharidoses and related disorders. Dentomaxillofac. Radiol., 1992, Vol. 21, 4549, February.

6. Tamer Zerener, Gurkan Rasit Bayar, Hasan Ayberk Altug, and Serkan Kiran. Extremely Rare Form of Impaction Bilateral Kissing Molars: Report of a Case and Review of the Literature. Case Reports in Dentistry vol. 2016, 2560792, 5 pages. 